\title{
DE90 000685
}

H'L liFXFRA. MAY-RODY SYSTPS

ajomons, S. A. Trukenn, T-II

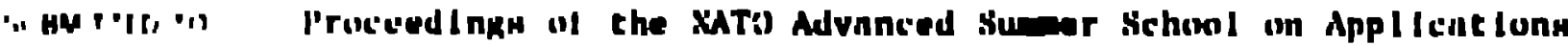

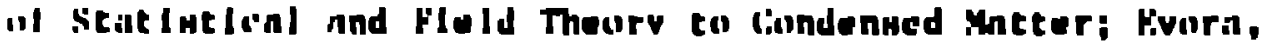
portugnI: Miv, I4R4

\section{IMER TAIMR:P}

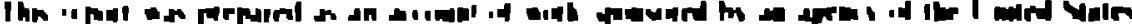

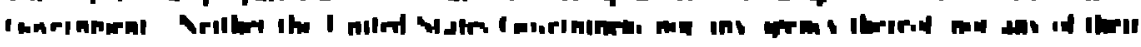

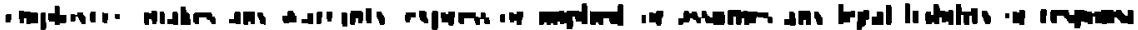

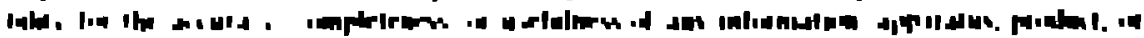

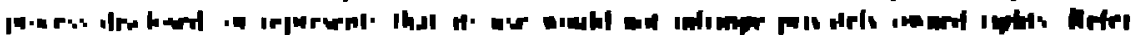

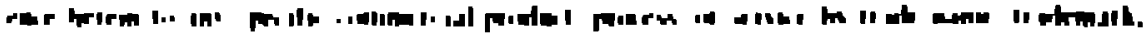

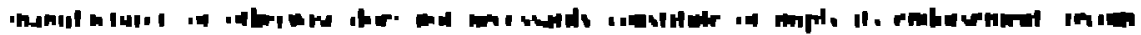

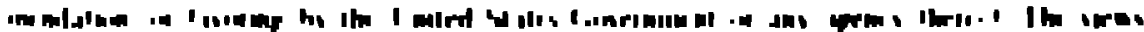

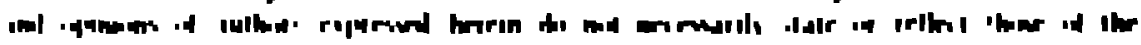

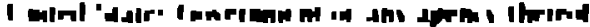

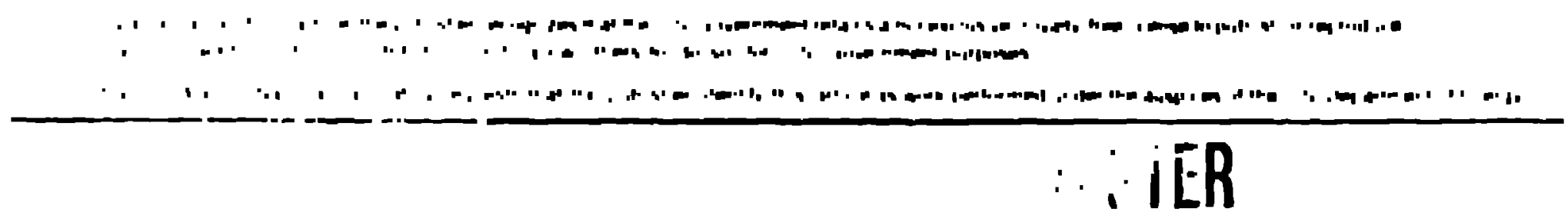

$\|$ (C) (S) A 
GENERAL MANY-BODY SYSTEMS

\author{
S. A. Trugman \\ Theoretical Division \\ Los Alamos National Laboratory \\ Los Alamos, NM 87545
}

\title{
INTRODUCTION
}

The problem nf how to visualize and sometimes solve a general many-hody system is considered. The ideas are established in the context of very simple small systems. a Hubbard model and a coupled electron-phonon model, both on two lattice sites. These models ane also solved to good approximation in the themodynamic limil, although the Hubbard model is restricted in a small number of holes away from the Mott insulating state. Response functions are also conside.ed.

A fairiy general many thxdy Hamilionian is

$$
H=H_{1, a l}+H_{a 1-a l}+H_{a l-p h}+H_{p h} \text {. }
$$

c'onsisting of an electmon of other fermion kinetic energy and electron-electron interactions, which may he coupled to a bose field such as a phonon The phonons themselves may he nonlinear (have self-imteractions) The system may be strongly coupled $\left(H_{f}, d\right.$ and $H_{d}$ ph may he large $)$. (ne may also aud coupling to an extemal driving lield, surh as an AC electric field. line methxds disceusised are nonperturhative, and so tiffer from the standurd medinxl: of diapranmmatic

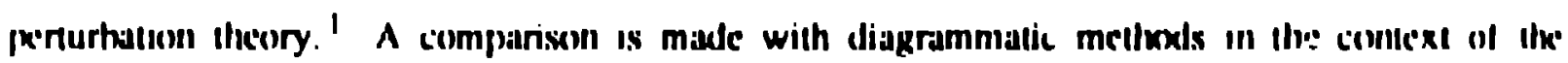
ralndonn phisese apnnoximalicm 


\section{HUBBARD MODEL (SMALL SYSTEM)}

The tirst example is the Hubbard model, which describes interacting electrons and contains only the tirst two terms of Eq. (1):

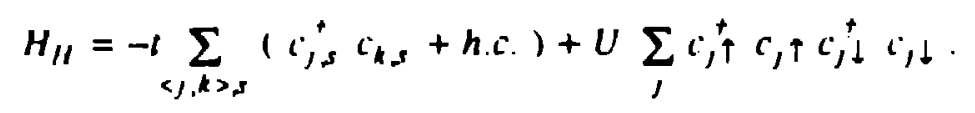

The operator $c_{j, s}$ creates an electron of spin $s$ on a Wannier orbilal on lattice site $j$. The first term (clectron kinetic energy) causes electrons to hop to nearest neighber sites without changing their spin. The last term is a repulsive on site electron-electron interaction. To illustrale the exact solution of Eq. (2) for a small system, consider the problem with two sites, two elecimns, and the 1-component of the spin $S_{z}$, which is conserved, equal to zero. (Infinite systems will be considered laler.)

The Hilber space in coordinate representation is given by

$$
\begin{array}{ll}
1>=\uparrow & \downarrow \\
12>=\uparrow \downarrow & \\
1>= & \uparrow \downarrow \\
1>= & \uparrow .
\end{array}
$$

where the lisst sile is on the left and the second on the right. The Hamilionian operaling on il connects (has nonzero matrix elements) to stules $|2\rangle$ and !3>. State $\mid 4>$ also connects $10|2\rangle$ and 3), see Fig. 11). The diagonal energy of states $\mid 2>$ and $\mid 3>$ is $U$, and that of $\mid 1>$ and $\mid 4>$ is 0 .

Note that an interacting many-body problen (containing the product of fold fermion operaltors) has been mapped onto a non-enteracting one panticle tight-hinding problem. If the

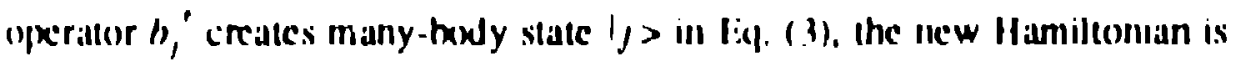




$$
\dot{H}=\sum_{, k} i_{j k}\left(b_{j}^{+} b_{k}+h(i)+\sum_{j} \varepsilon_{1} b_{j}^{+} b_{j} .\right.
$$

with no interactions (four-fermion operators). The sites in the tight-binding problem, however, represent many-body states, not the usual atomic or Wannier orbitals. It is a general r.esult that one can always exactly map thi ground and excited states of an interacting many-body problem onto those of a noninteracting one-body problem in this way.

In matrix notation, $\dot{H}$ is

$$
\tilde{H}=\left[\begin{array}{cccc}
0 & -t & -t & 0 \\
-t & U & 0 & -t \\
-t & 0 & U & -t \\
0 & -t & -t & 0
\end{array}\right]
$$

This matrix is simple to diagonalize exactly. The four eigenvalues are

$$
\left.\left(E_{1}, E_{2}, E_{1}, E_{4}\right)=\left(\left(U-\sqrt{U^{2}+16 t^{2}}\right) / 2,0, U,: U+\sqrt{U^{2}+16 t^{2}}\right) / 2\right)
$$

The two lowest energy (unnormalized) eggenvectors are

$$
\begin{aligned}
& \left.: \psi_{1}\right\rangle=|1\rangle+|4\rangle+a(|2\rangle+\mid 3>) \\
& \left.\left.: \psi_{2}\right\rangle=|>-| 4\right\rangle .
\end{aligned}
$$

where $a=-E_{1} / 2 t$. i $\left.\psi_{1}\right\rangle$ is a singlet state and $\mid \psi_{2}>$ is the $S_{2}=0$ iriplet state. For $U>t$. the low energy part of the Hilber space is described by

$$
H=\operatorname{unst}+J \sigma_{1} \sigma_{2}
$$

with $J=t^{2}, U$.

It appeas that the singlet and triplet states are written with incorrect signs. The signs are in fact correct, which brings up an issue that was glossed over: the ordering of anticommutung lemion operatos. For the problem above, the relerence ordenng is to have the up spuns ofx rate IITY. 


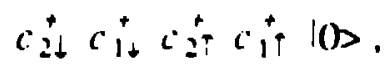

where $i(>$ is the vacuum. A state with a positive sign is given by operators in the above order, for example $: 2>=c_{i \downarrow}^{+} c_{i \uparrow}^{+} \mid 0 s$. Suppose one had chosen a different ordering convention. such as putting sitc I operators first:

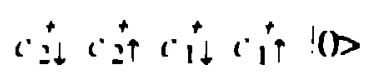

The new light binding model is shown in Fig. (2). The $2-4$ bond sign, for example, is obtained with the convention of Eq. (7) as lollows:

$$
H_{1}|\dot{2}\rangle=-t\left(c_{2 \uparrow}^{+} c_{1 \uparrow}\right) c_{1 \downarrow}^{+} c_{i}^{+}\left|(\mid)=+t c_{2 \uparrow}^{+} c_{i \downarrow}^{+}\right| 0 s=+t|4\rangle \text {. }
$$

The cigenvalues of the new problem (Fig. 2) are the same as those of Fig. 1. and the wavefunctions are "covariant": $\left(\Psi_{1}, \psi_{2}, \psi_{3}, \Psi_{4}\right)_{1} \rightarrow\left(\Psi_{1}, \Psi_{2}, \Psi_{3},-\Psi_{4}\right)_{2}$. Now a singlet is written in the conventional way.

This is in fact a lype of gauge transformation. An example of a general tight-binding model is given in Figure (3). If one changes the tefinition of a basis state (e.g. $\left|\phi_{1}\right\rangle \rightarrow-\left|\phi_{1}\right\rangle$ ). all of the bx)nds coming from $\left|\phi_{1}\right\rangle$ change sign, as shown in Figure (4). In general any loop with an even number of $+l$ bonds may be trunsformed into a loop with no $+f$ londs by a suitable choice of gallge (sign ol basis functions). All +t honds may also be removed from bonds that are not pirn iff a loxp. such as 8-9. Hov'ever. loops with an odd nurnter of to honds are frustratced the $+t$ honds may not be gauged away). The gauge transfomation generalizes to $\phi_{1}>\rightarrow e^{1+\mid} \mid \phi_{1}>$. uhere $\theta$ was biken equal to $\pi$ above.

Similar issues arize in the yuantum ltall effect when a magnetic lie'd penetrates a lattice. In That case a nux through a lesp that is an integer times the flux quantum $\Phi_{0}$ is the samte as c(n) llux under a gauge iranstomation. 
One can write down an approximate ground state of an unfrustrated tight binding model almost by inspection. First gauge transform away all $+t$ bonds. Then all the $\psi$, have the same sign in the groundstate. with $\psi$, larger on sites that have a lower energy, and more or larger connected $t_{1}$, bonds.

One can do larger Hubbard models exaculy. For example, with $2 \mathrm{~N}$ sites and $2 \mathrm{~N}$ electrons, half of which are spin up, one must diagonalize a matrix of size $\left(\frac{2 N}{N}\right)^{2}$ on a side. Six sites yicld a $400 \times 4(x)$ matrix, or equivalently a tight-binding model with $4(x)$ sites. which can be diagonalized completely on a computer. Ten sites yield a $63,504 \times 63.504$ matrix, which can be solved for the ground and low lying excited states by the Lanczos method. 2 There is no exact solution for the infinite Hubbard model.

\section{POLARONS (SMALL SYSTEM)}

The second example is a coupled electron-phonon system. described by

$$
\begin{aligned}
H=-r & \sum_{<, k>, s}\left(c_{j, s}^{+} c_{k, s}+h_{c}\right)+U \sum_{j} n_{j} n_{j}+V \sum_{<, k, j} n_{j} n_{k} \\
& +\lambda \sum_{j}\left(n_{j}++n_{j}\right)\left(a_{j}+a_{j}^{+}\right)+\Omega \sum_{j} a_{j}^{+} a_{j} .
\end{aligned}
$$

where $n, \uparrow=c ; \uparrow c, \uparrow$ and $n_{j}=n, \uparrow+n, \downarrow$. In the $1-d$ version, electrons nun along a chain. possibly interacting with each other on site and on nearest neightor sites ( $U$ and $V$ terms respectively). Fach site is coupled to a hamnonic oscillitor, so that the oscillator leels an extra force when an clectron is on that site, $\delta H=-\lambda_{1} x$, where $x$ is the phonon coxordinate. In terms of the creation operator a' for the oscillator.

$$
P=\sqrt{2 m \omega}\left(a+a^{*}\right)
$$

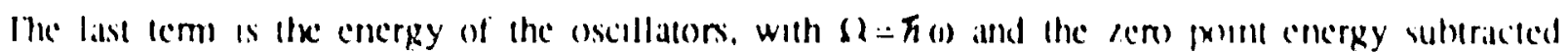


off. This model is for a phonon energy that is independent of $\vec{k}$, or optical phonons. (If $a_{j}^{*} a_{k}$ lerms were added to the Hamiltonian, the phonon energy would have a nonzero dispersion.) The Hamiltonian in Eq. (8) describes the system shown in Figure (5).

For simplicity. consider first a two site problem with 1 electron and two ohonons. With only one electron present. the $U$ and $V$ lerms do not operate. The basis functions can be laken cither in position or momentum space. For varicty, and to compare with the random phase approximation (RPA), the calculation will be done in momentum space. For a lwo sile lattice, only $\vec{k}=0, \pi$ are allowed.

There are 2 electron basis states.

$$
\begin{aligned}
& 0_{e l}>=\frac{1}{\sqrt{2}}\left(\left|1_{e l}>+\right| 2_{e l}>\right) \\
& \pi_{e l}>=\frac{1}{\sqrt{2}}\left(1_{e l}>-\mid 2_{e l}>\right)
\end{aligned}
$$

The energy of the first is $-t$ ard of the second is $+f$. There are altio two phonon creation operalors

$$
\begin{aligned}
& a_{0}^{+}=\frac{1}{\sqrt{2}}\left(a_{i}+a_{i}^{*}\right) \\
& a_{n}^{*}=\frac{1}{\sqrt{2}}\left(a_{i}+a_{2}^{*}\right)
\end{aligned}
$$

liach a can create arbitrarily many quanta. A many-body stute is specified by

$$
y_{e l}>\ln _{1}>\operatorname{in}_{\pi}>
$$

where $y_{1}=?$ ) or $\pi$, and the phonon occupation numbers are $n_{0}=0,1,2, \ldots, n_{n}=0,1,2 \ldots$. The dectron phonon interaction conserves momentum. Its strength is momentum independent in this modal 
The equivalent I-body tight binding model consists of two disconnected pieces for this model, one for each total momentum. The total momentum $K=\pi$ piece of the Hilbert space is shown in Figure (6). The $q_{e l}$ can be deduced from the total $K, q=\left(K-\pi n_{\pi}\right) \bmod 2 \pi$, so by specifying the phonon state, one also specifies the electron state. The lowest row of vertices has $q_{e l}=\pi$, ctc.

The diagonal energy of a site is

$$
E\left(n_{0}, n_{n}:=\Omega\left(n_{0}+n_{\pi}\right)+(-1)^{n_{n}} \quad(K=\pi) .\right.
$$

The numerical factors in the off-diagonal matrix elements can be obtained using $u^{+} i n>=\sqrt{n+1} \mid n+1>$. The encrgy in Fig. ( $(6)$ therefore increases linearly to the upper right, with a corrugation as a function of $y$. In this basis, $r$ appears in a diagonal (sice) energy, in contrast to the real-space basis in the previous example, where $t$ is an off-diagonal bond strength. There is an identical lattice for the $K=0$ sector, except that the $y$ corrugations are oppesite.

$$
E\left(n_{0}, n_{n}\right)=\Omega\left(n_{0}+n_{n}\right)-(-1)^{n_{n}} \quad(K=0) .
$$

To lind the ground state and low lying excited states numerically, one truncales the lattice (keeping states to the lower left. with low diagonal energies). The remaining problem is solved numericully. One should check that the truncation does not effect the physics, by verifying that : wavefunctions and energies of the low lying eigenstates have converged.

The interaction with the phonons is said to be retarded or frequency-dependent. In this fomulation, however, one need not include an explicilly frequency-dependent interaction, but merely couple in phonon states of various energies on an equal footing with all other stutes.

How do standard diagrammatuc methods, such as the random phase approximation (RPA). compare with solving the equivalent light-binding latlice"? Consider the question of loww the $k=\pi$ phorion energy is changed by the electenn-phonon interaction. If $\lambda$ were $z$ ero, the hare $k: \pi$ 
phonon would be an eigenstate of energy $-t+\Omega$. This is the state $\left(n_{0}, n_{\pi}\right)=(0,1)$ in Fig. (6). The RPA sums all diagrams of the form shown in Figure (7), with an:y number of bubbles. The verical line on the left cuts through the siate with one $k=\pi$ phonon and no electron-hole pairs, which is state $(0.1)$ in Fig. (6). The verical line on the right euts through the state with an electron-hole pair and no phonons, which is the state $(0,0)$. In the exact problem, there are also matrix elements from state $(0, i)$ to $(0,2)$ and to $(1,1)$. These matrix elements are the verices shown in Figure (8), which are neglected by the RPA.

The RPA thus keeps only the two states $(0,1)$ and $(0.0)$ and the bond between them, and throws away the rest of the lattice, as shown in Figure (9). It solves this tiny "two site" problem exactly. When is this a good approximation? One requires $\lambda<t$ for there to be no significant admixtures of the neglected state $(0,2)$ in the ground state. Furthermore, $\lambda<\Omega$ is required to prevent signilicant admixtures of state $(1,1)$, which was also neglected. In this limit however, the hare phonon state $(0,1)$ is essentially exact, so that one need not have bothered with more than one state. The RPA is this not very useful for this case.

Various response functions (Green's furctions) can be calculated direcly from the eigenstates. For example the optical absorption is

$$
\alpha(\omega)=\frac{1}{\omega} \sum_{n}^{\prime}<n\left|j{ }^{\prime}()\right|^{2} \delta\left(\omega-\left(\varepsilon_{n}-\varepsilon_{()}\right)\right)
$$

where $j$ is the current operator. If the Hilbert space is truncated. $\alpha(\omega)$ becomes unreliable for very large (1).

\section{I.AR(;F. SYSTEMS}

A general large many-body problem cannot be solved by any methexd, including this one. Consider the liubhard model with $1\left(t^{23}\right.$ siles at artitrary filling in the momentum space hasis. In 
this treatment, the lirst state (noninteracting fermi ground state) connects via $L$ to an enormous number of states $O\left(10^{(\omega)}\right)$. . ie problem at this level, which is a very large "star" light binding model (Fig. 10a), is stial straightforward to solve exactly. However, there is no justification tor stopping at this level. Each perimeter state connects to a large number of other states, sometimes forming loops, and each of them connects to many new states, etc., so the problem finally becomes intractable. This is illustrated schematically in Fig. (10b). The many-body problem has still been mapped exactly onto d one-body tight-binding problem, but one that is too large to solve.

There is, however, a class of problems that can be solved essentially exactly or to good approximation on an infinite lattice. These problems describe the quantum dynamics of one or several "defects" in a weli-understood background. Two examples are: (1) The problem in which a small number of electrons interact with optical phonons to form polarons, bipolarons, etc. on an infinite lattice. (2) The prot!em of holes and pairs of holes in the Mott insulating state of the Hubbard model on an infinite lattice in two or more dimensions.

The polaron problem is the same electron-optical phonon problem described atove, but done on an infinite lattice in real space rather than $k$-space. The many-bociy basis states are

$$
\left.j>n_{j}>n_{j+1}>\left|n_{j-1}>i_{j+2}>\right| n_{j-2}\right\rangle
$$

The tirst ket is the electron location, followed by the number of phonons on the same site, on nearest neighbor siltes. etc. Again one consinucts an arbitrarily large variational space, and then checks that the space is big enough.

A small variational space might allow for zero or one phonon on the site that tine slectron is on or on a neare:t neighbor site. (A much larger space is used for accurate ....uculations.) The imall variatuonal space can be written 


$\begin{array}{cccc}\text { state } & -1 & 0 & 1 \\ 1 & 1 & 0 & 0 \\ 2 & 6 & 1 & 0 \\ 3 & 0 & 0 & 1 \\ 4 & 1 & 0 & 0 \\ 5 & 0 & 1 & 1 \\ 6 & 1 & 1 & 0 \\ 7 & 1 & 0 & 1 \\ 8 & 1 & 1 & 1\end{array}$

The headings: show the number of phonons on the site to the left of the electron $(-1)$, on the sume site as the eiectron (0), and to the right of the electron (1). All translations of thes: states are alwo Sluded in the Hilben space. The tight-binding lattice is shown in Fig. (11). The vertical honds have strength $-\lambda$, and the others strength $-t$. The diagona! energy is zero for state $1, \Omega$ for states 2. 3. and 4 , and $2 \Omega$ for states 5 and 6 . Different states with the same number represent translations of a state. For example. the leftmost state 3 represents the state with an electron on site 0 and a phonon on site 1 . The middle state 3 represents the state with an electron on site 1 and a phonon on site 2. States 7 and 8 form a disconnected part of the Hilber space and are not shown. There are $6 N$ states in the Hilbert space. where the number of sites $V$ is inlinite. The Hilben space is translation invariant, with 6 states per unit cell. The translation invariance implies tha! the exact eigenstates obey Bloch's theorem. For any crystal momentum $\vec{k}$. one need diagonalize only a $6 \times 6$ hermitian matrix. rather than a $6 N \times 6 . N$ matrix.

The ground state eigenfunction of the :ight-binding model describes a polaron. Note that even with this small Hilbart space, there is already more than one way to propagate through the latuce. wther directly along the baselit. or through a high loop. The loop mute shows that the pxlarnn can move by making a virtual intemal excitatioti, and then getting rid of it. Different propagauon rnutes interfere constructively in the physical polamn. The polaror is never "self trapped." but rather is delocialized in a Bloch state of wavevector $\dot{k}$. possibly with a large effectuve mass. 
Plotting the tight-binding eigenvalues as a function of $\vec{k}$ gives a graph that looks like a band structure, although it describes many-body physics. The lowest band is the polaron quasiparticle energy $\varepsilon(\vec{k})$ for the electron dressed with phonons. The higher bands are either excited states of the quasiparticle or unbound electron-phonon states. One can examine how the quasiparticle energy, wavefunction, and residue $Z$ vary with $k$. Figure (12) shows these bands for a larger variational Hilbert space that includes 100 variational states per lattice site. Other calculations with several thousand variational states, involving many phonons in a large neighborhood of the lectron, have been performed. Calculations have also been done for hipnlarons, nonlinear phonons, and for the AC conductivity of a polaron. ${ }^{3}$

The same method can be applied to w. ar many-body froblems on an infinite tattice. The Hubbard medel in two dimensions, with one electron per site forms a Mott insulating state with antiferromagnetic long-range order. ${ }^{4}$ The problem of one and two holes in the Mott insulating state and the interaction between the hole has been studied. "In this case the variational space consists of the location of the hole(s), and a number of spin-flips relative to the Neel state in the vicinity of the hules. The Green's function for hole propagation, which contains excited state information, has also been oblained. ${ }^{6}$ These studies have used a variational space as large as $6(19$ states per real space lattice site.

\section{SIMMARY}

for a small system, one can solve essentually exactly for the low-lying eigenvalues. cigenfunctions, and lirkat response to an extemal probe. These systems maly include clectronclectron, electron phomon, and nonlinear phonon interactions. Standard dagrammatic technicques. like the RPA may be madeciuate for the:a problems. Some intinite systems can be solved exilciny or to goed appoximatuon hy the same technique, which exactly maps a many body problem onto 
a one-body ti,ght-binding model.

I would like to acknowledge I. Batistic and S. Marianer for their substantial contributions to the polaron sections. This work was supported by the US DOE. I would also like to thank the ITP at Santa Barbara for their hospitality, and their additional support under the NSF Grant PHY 82-17853, supplemented by funds from NASA.

\section{References}

(1) Sce, c.g., A. A. Abrikosov, L. P. Gorkov, and I. E. Dryaloshinski, Methods of Quantum Field Theory in Statistical Physics (Pergamon, Elmsford, NY, 1765), S. Doniach and E. H. Sondheimer, Green's Functions for Solid State Physicists (Benjamin, Reading, MA, 1974), A. L. Fetter and I. D. Walecka, Quantum Theory of Many Particle Systems (McGraw-Hill, NY, 1971), (i. D. Mahan, Many-Par.icle Physic's (Plenum, NY', 1981).

(2) S. Pissancleky, Sparse Matrix Trithology (Academic, NY, 1984).

(3) I. Batstic, S. Marianer, and $S$. Trugman, unpublished.

(4) J. D. Reger and A. P. Young. Phys Rev, B .17, 5978 (1988).

(5) S. A. Trugman. Phys Rev. B 37. 1597 (1988).

(h) S. A Trugman. I.ANI prepnne 892230

\section{Figure Captions}

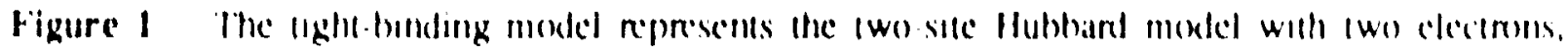
5, 1). The hends are ofl diagonal matnx cicments of amplitude 1 
Figure 2 The modified tight-binding model for the two-site Hubbard model, using the ordering convention in E4. (7). The slashed bonds are off-diagonal matrix elements of amplitude $+t$, and the unslashed bonds of amplitude $-\cdot$.

Figure 3 A general tight-binding model may contain loops, dead ends, and sites with different coordination numbers.

Figure 4 A gauge transformation of the previous tight-binding model accomplished by $\phi_{1}>\rightarrow-\mid \phi_{1}>$. The slashed bonds are matrix elements $+r$.

Figure 5 A polaron system in which electrons hop along a chain. Each site on the chain is associated with a harmonic oscillator. If an electron, represented by an arrow, is present on a site, an additional force is applied to the oscillator on that site.

Figure 6 A portion of the infinite tight-binding model representing a coupled electron-phonon system. The sector pictured has total momentum $K=\pi$. A state (site) is labeled by $\left(n_{0}, n_{\pi}\right)$. where $n_{0}$ is the number of momentum zaro phonons and $n_{\pi}$ is the number of momentum $\pi$ phonons. The dectron nomentum $y$ is shown on the right for each ow. The honds are off. diagonal matrix elements of amplitude $\lambda$ times a numerical constant.

Figure 7 A tiagram retained in the RPA approximation.

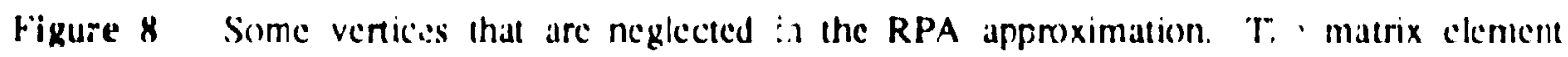
connecting state $(0,1)$ to state $(0,2)$ is shown diagramatically above, and the one connecting $(0,1$, (1) 11.1111 shown below.

Figure $y$ for this problem, the RPA retans only the bond and Iwo sites that are shown in Wiak. discarding the rest of the intinte lattice.

Figure 10 (a) The large "star" obtaned as the first approximatuon to the slubbard model in the

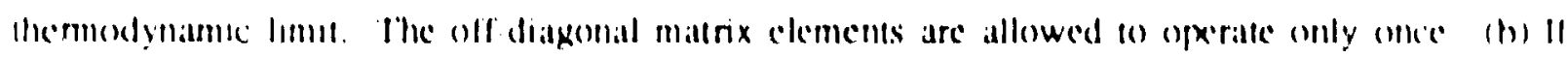

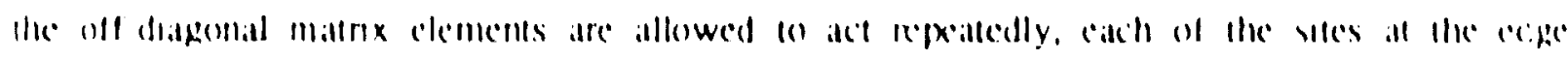

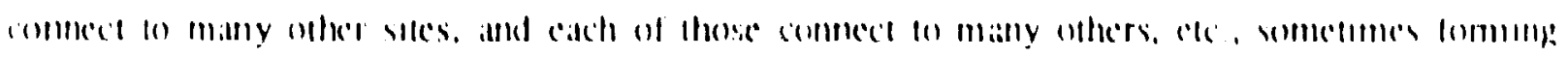


loops. (Shown schematically.)

Figure 11 The tight-binding model for a polaron on an infinite lattice. With the small basis set of Eq. (10), the tight-binding lattice extends to intinity and is periodic.

Figure 12 The cigenvalues of the polaron problem is plotled as a function of wavevector $k$. 


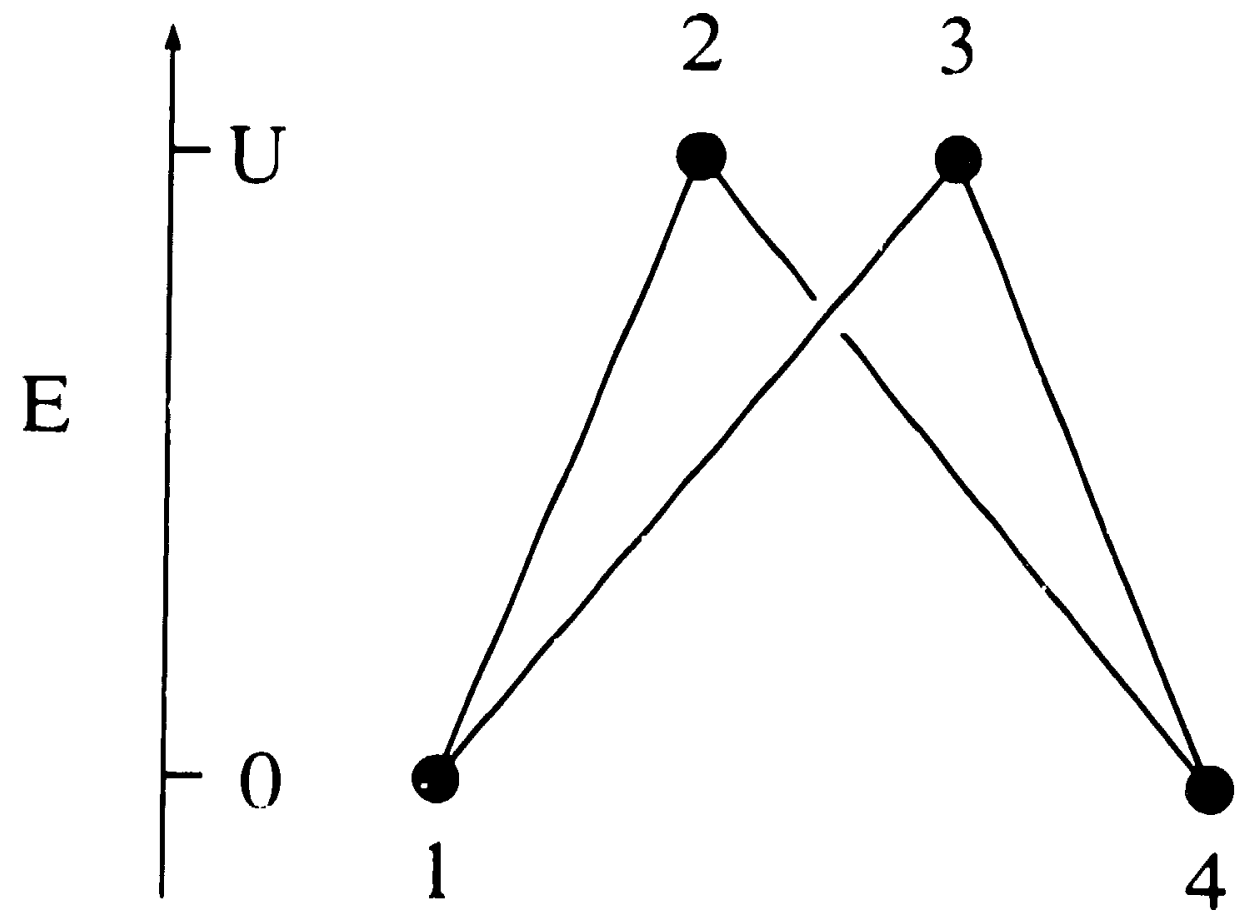

Fig. 1 


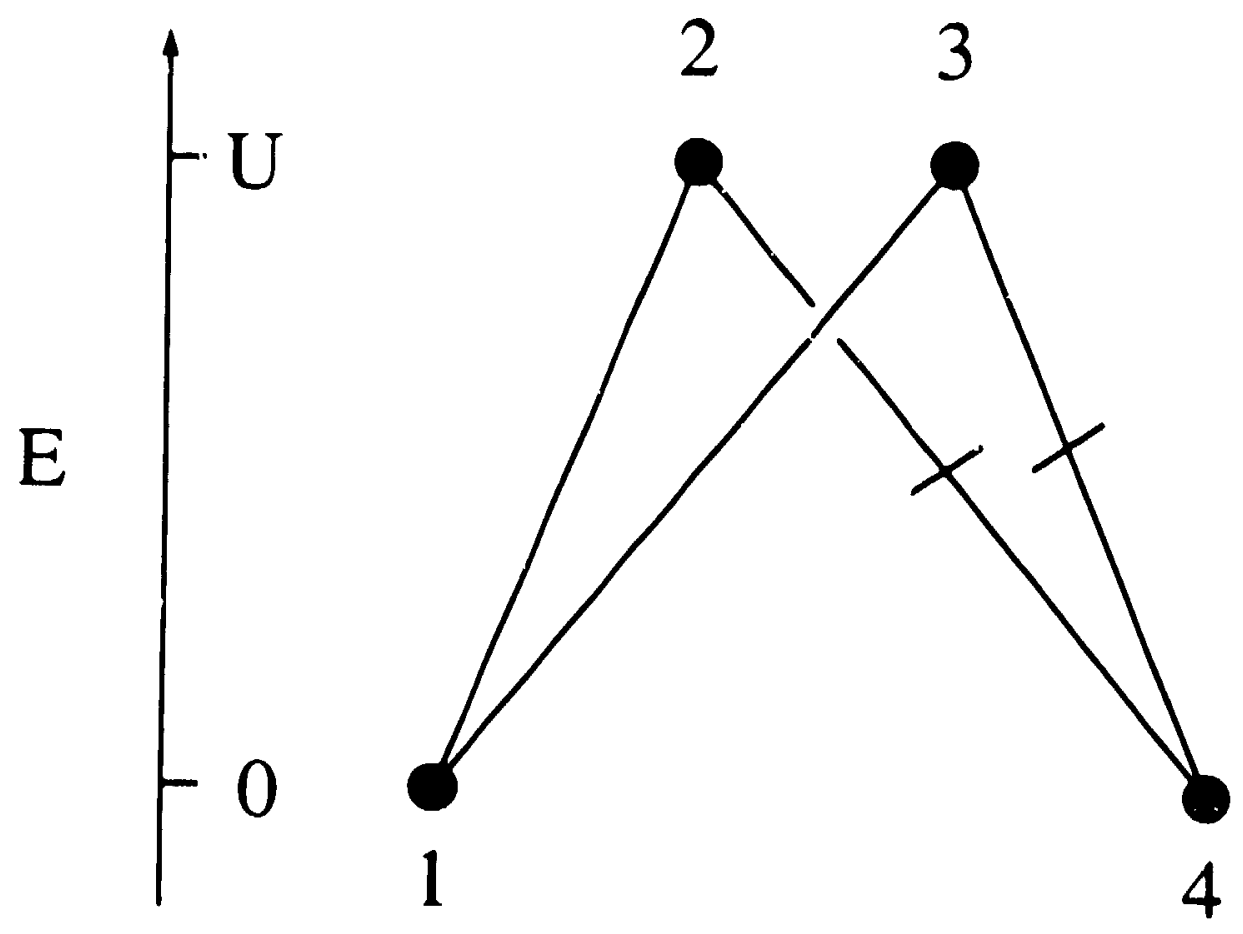

Fig. 2 


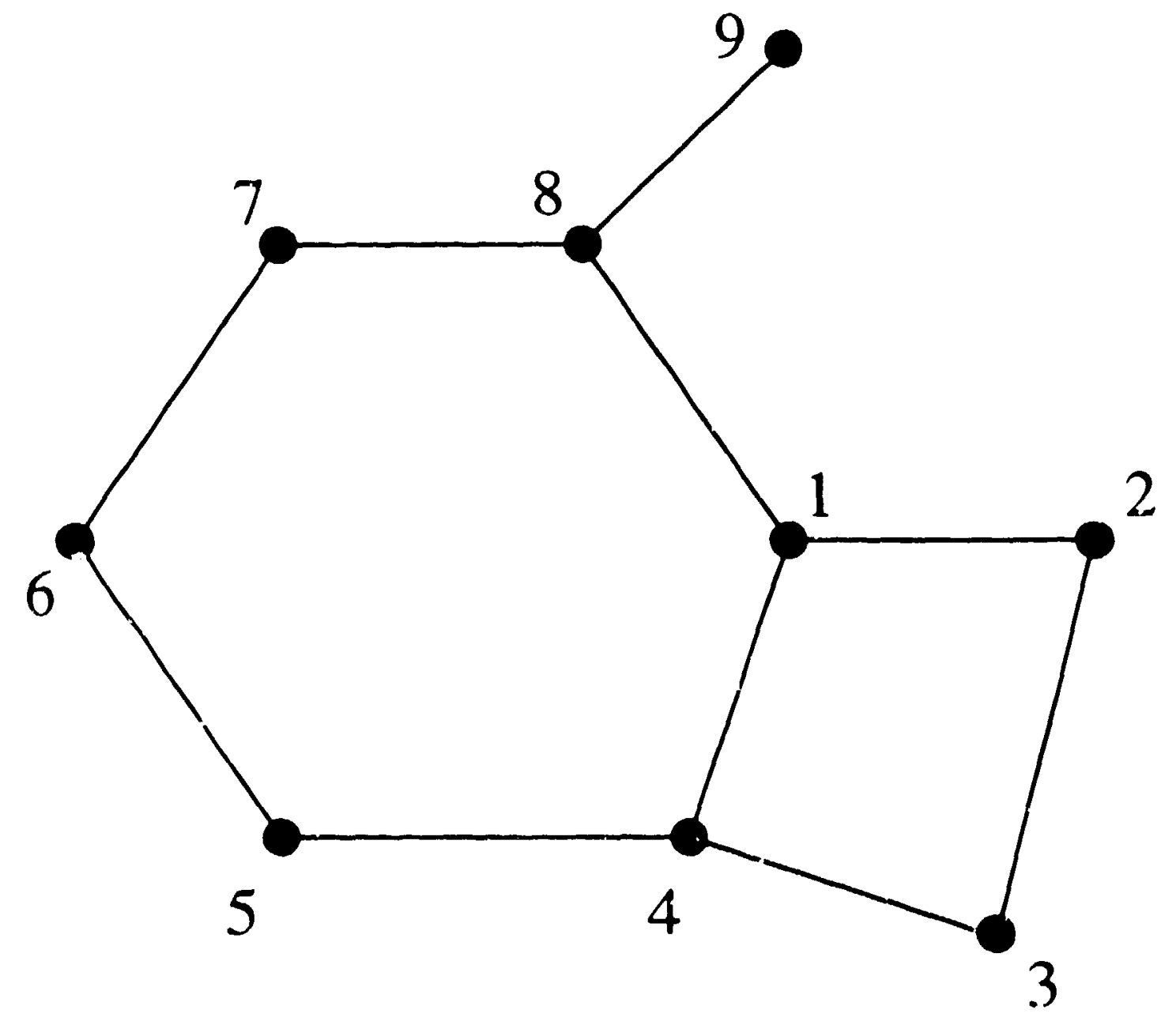

Fig. 3 


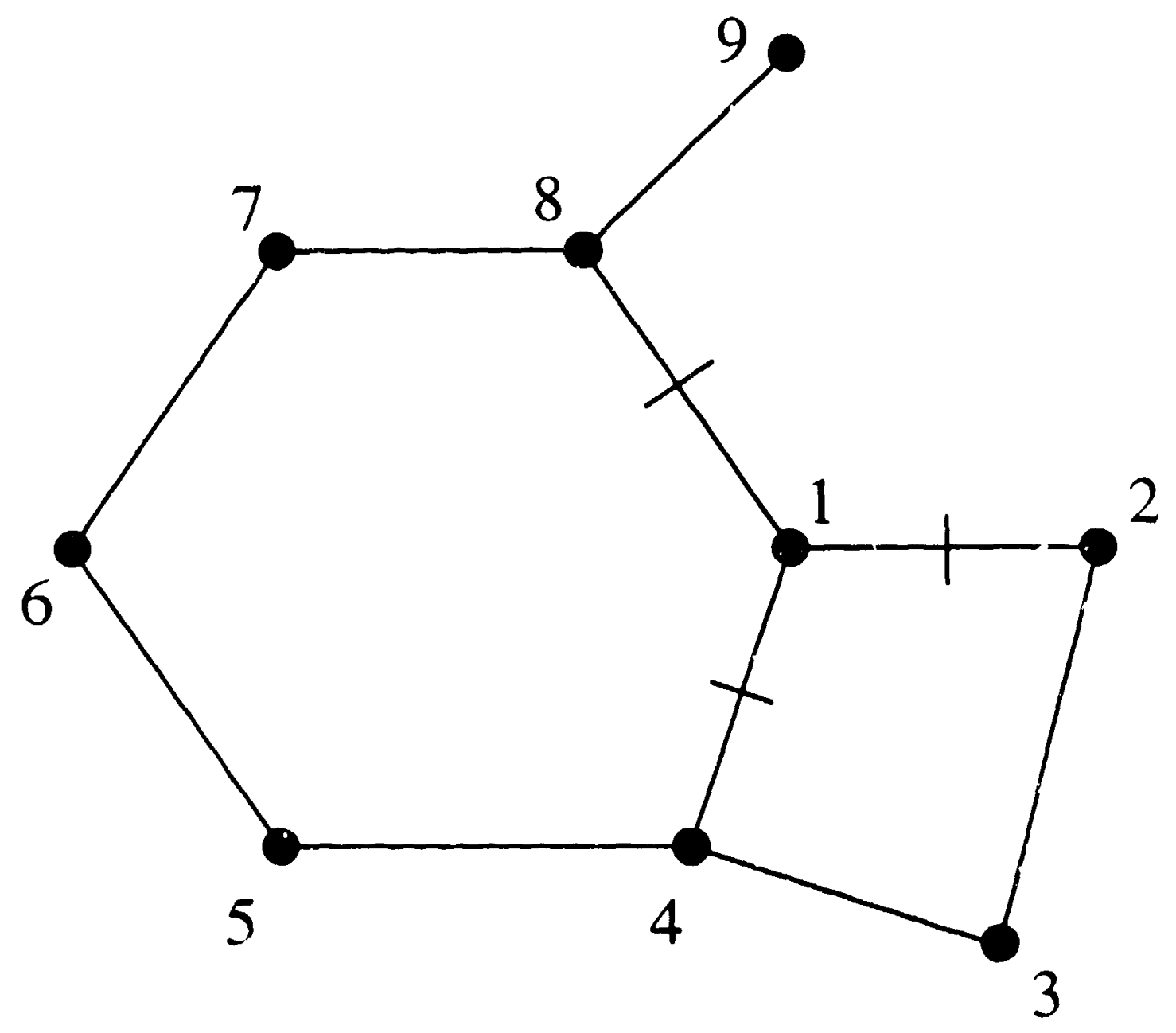

Fig. 4 


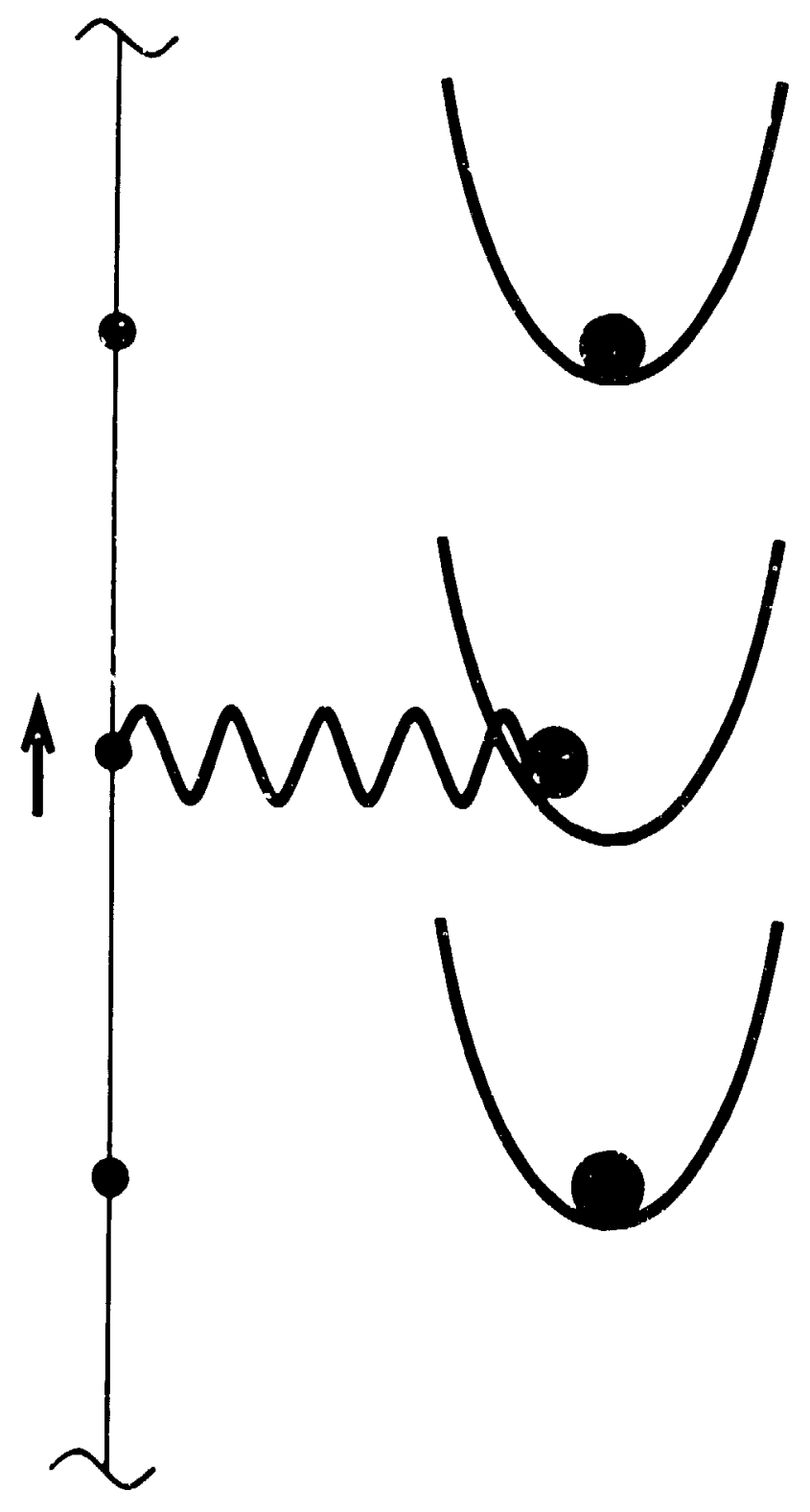

Hig. 5 


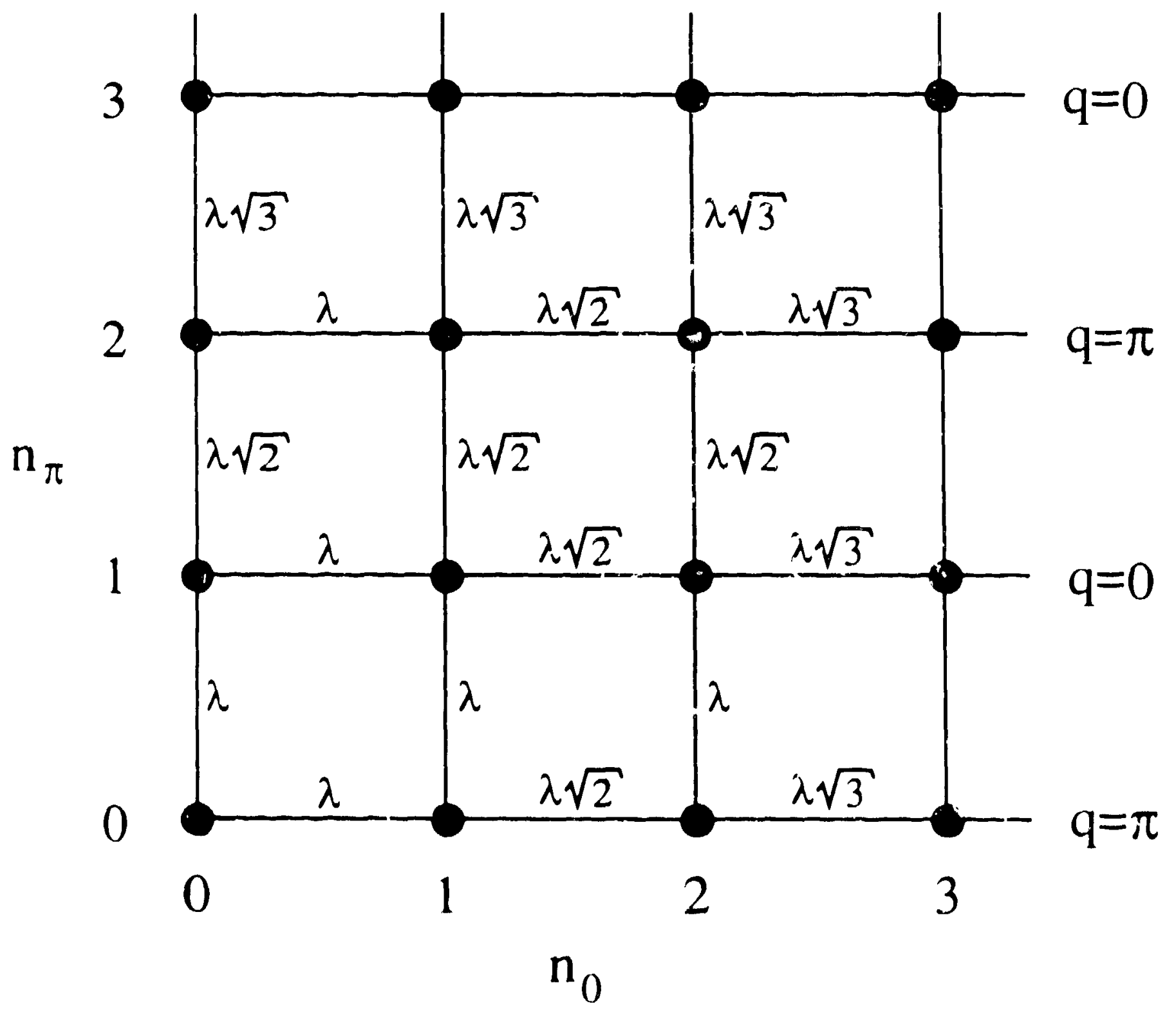

Fig. 6 


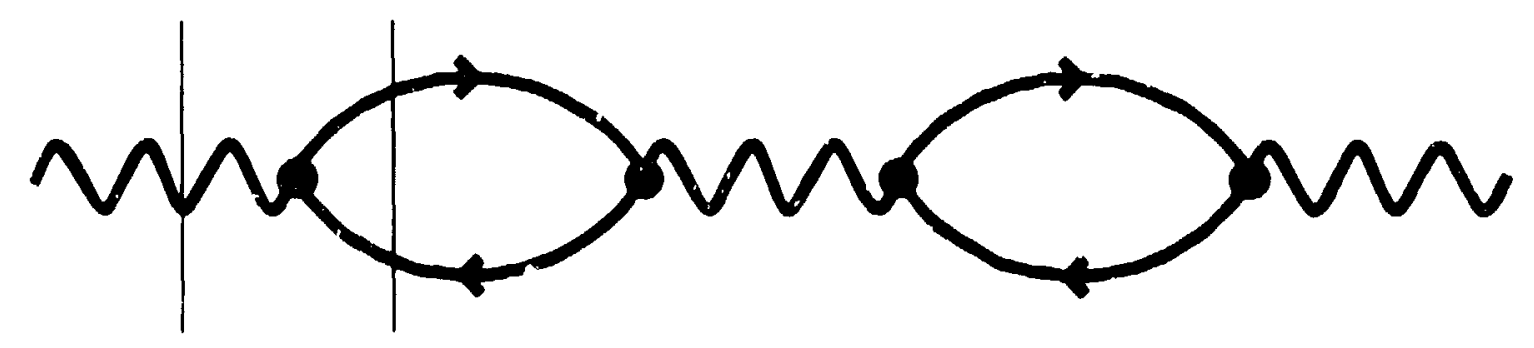

Fig. 7 

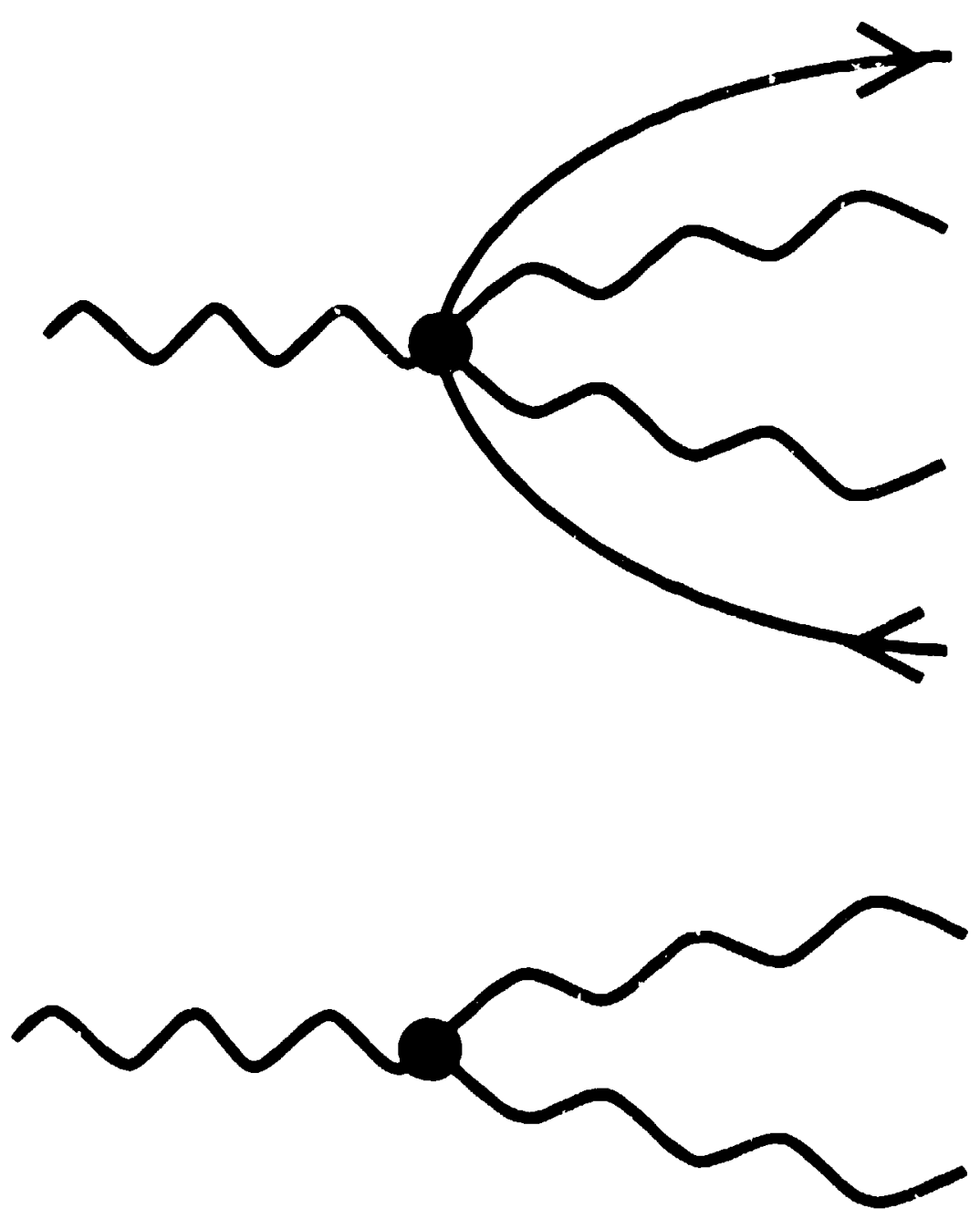

Fig. 8 


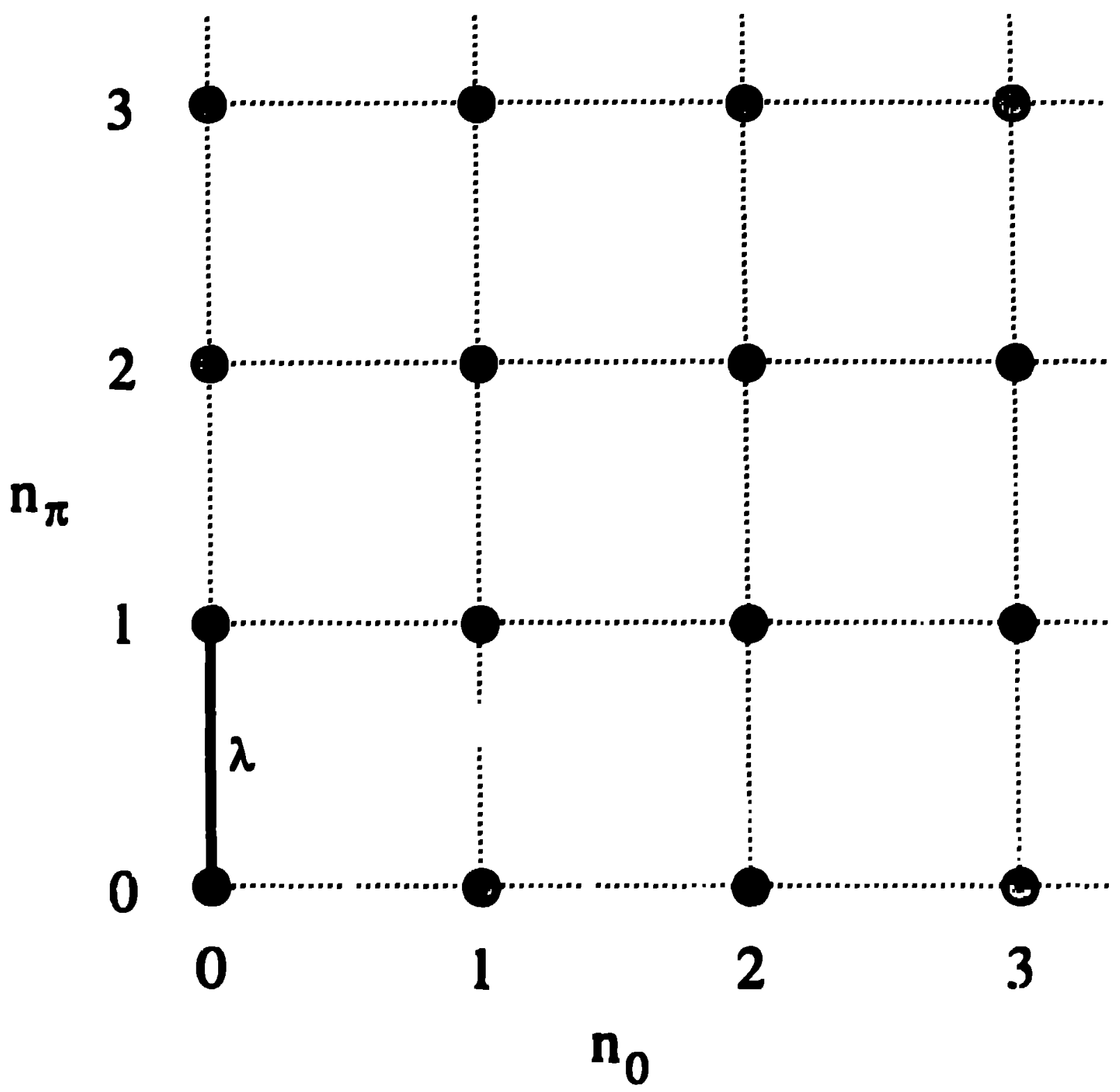

Fig. 9 


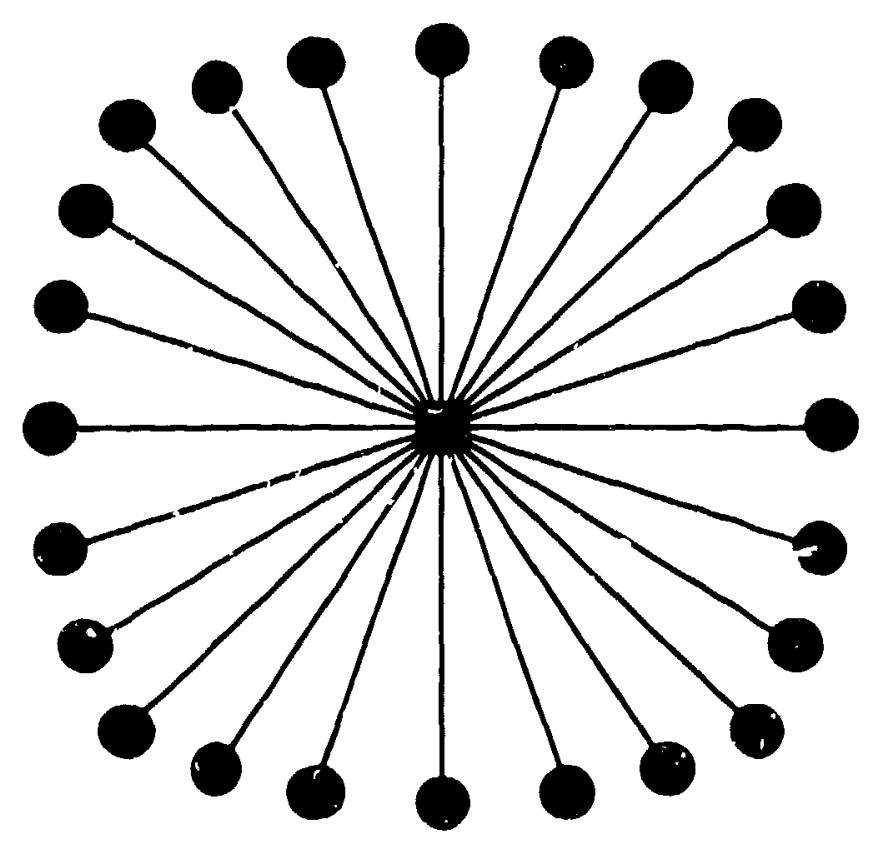

(a)

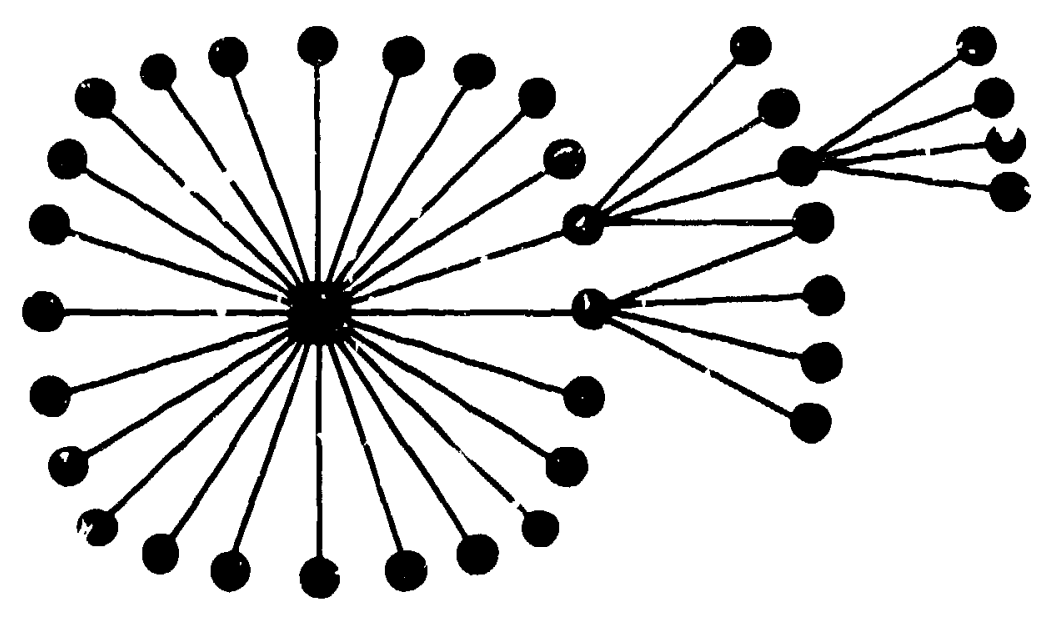

(b)

Fig. 10 


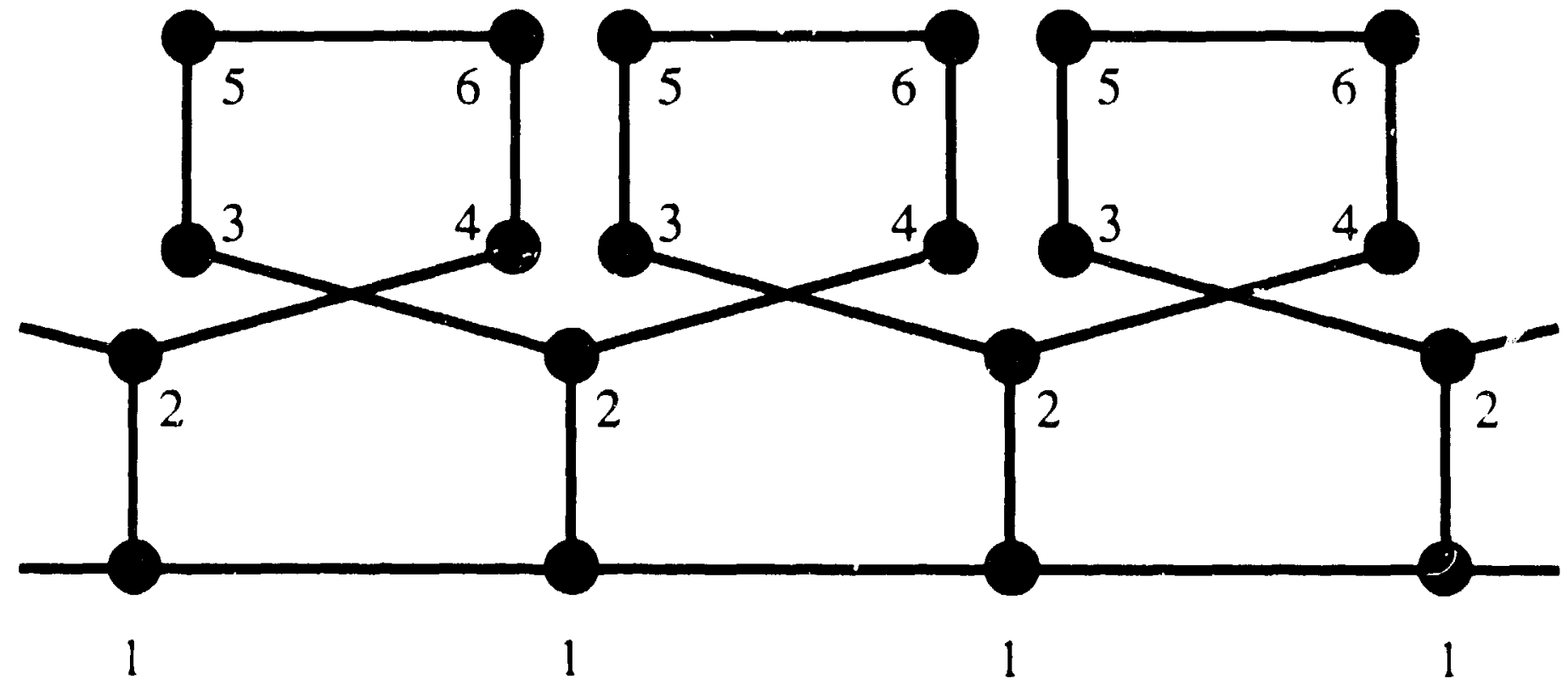

Fig. 11 
$:$ and I.cmbda- 1.001 .00 no. of phonone -3

nup -1

ndoun -0

no. of etalee-130

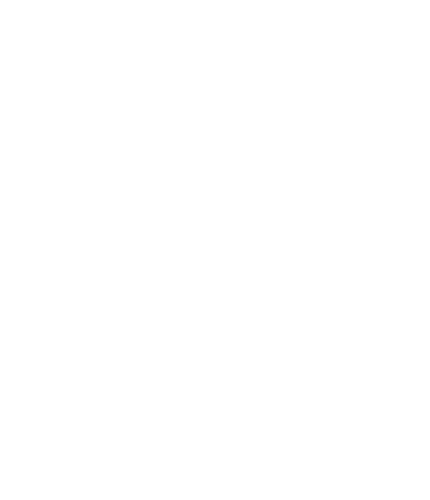

Fig 12

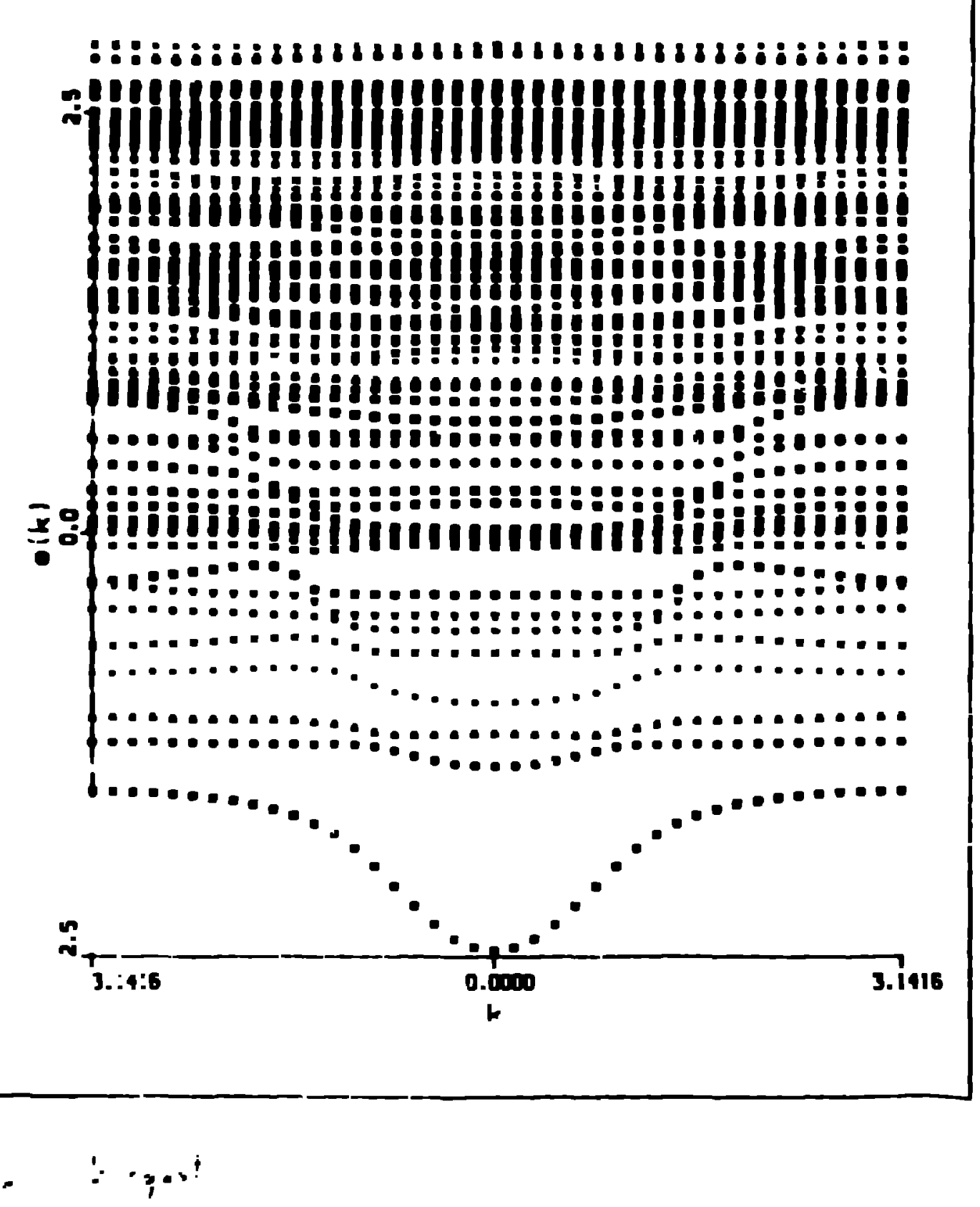

